\title{
Alteration of Anthocyanin Glycosylation in Cranberry Through Interspecific Hybridization
}

\author{
Nicholi Vorsa \\ Philip E. Marucci Center for Blueberry and Cranberry Research and Extension, Rutgers University, \\ 125A Lake Oswego Road, Chatsworth, NJ 08019 \\ James J. Polashock ${ }^{1}$ \\ USDA-ARS Fruit Lab, 125A Lake Oswego Road, Chatsworth, NJ 08019
}

ADDITIONAL INDEX WORDs. Vaccinium macrocarpon, Vaccinium oxycoccus, antioxidant, bioavailability, flavonoid

\begin{abstract}
The flavonoids of american cranberry (Vaccinium macrocarpon Ait.) are documented to be beneficial for human health. Among their benefits is a high antioxidant potential, with anthocyanin glycosides being the main contributors. Flavonoid glucose conjugates are reported to be more bioavailable than those with other sugar conjugates. The anthocyanin glycosides of $\mathrm{V}$. macrocarpon fruit are mainly galactosides and arabinosides of the aglycones, cyanidin and peonidin, with less than $8 \%$ glucosides. In contrast, the fruit anthocyanins of another cranberry species, $V$. oxycoccus L. were found to be largely glucosides of cyanidin and peonidin. Interspecific hybrids between these two species were intermediate to the parental species in the proportion of fruit anthocyanin glucosides. About half the progeny (1:1 segregation) in a backcross population (to $\mathrm{V}$. macrocarpon) maintained the relatively high anthocyanin glucoside ratio. In this study, we demonstrate the genetic manipulation of anthocyanin glycosylation in cranberry using interspecific hybridization, resulting in dramatically increased glucose-conjugated anthocyanins.
\end{abstract}

Flavonoids are considered to be secondary metabolites, which have been associated with roles in ultraviolet protection, plant sexual reproduction, pollinator attraction, symbiotic plant-microbe interactions, and plant-pathogenic microbe interactions (Koes et al., 1994). There is also a great interest in, and research being conducted on, the human health benefits of these compounds. Classes of flavonoid compounds, as well as specific flavonoids, have been identified as having particular bioactivity and/or health benefits (Nijveldt et al., 2001; Schijlen et al., 2004). Anthocyanins, for example, are considered to be potent antioxidants (Benvenuti et al., 2004). In addition, fruit of the Vaccinium L. genus, including the american cranberry, has been found to contain A-type proanthocyanidins, which inhibit adherence of P-fimbriated uropathogenic $E$. coli to uroepithilial cells, a necessary step in urinary tract infection (Foo et al., 2000a, 2000b).

Significant differences exist among flavonoids, including anthocyanins, in their antioxidant potential (Cao et al., 1997). Anthocyanins are typically conjugated to sugars, and antioxidant potential appears to be a function of both the glycoside as well as the specific aglycone, i.e., cyanidin, malvidin, pelargonin, etc. (Satue-Gracia et al., 1997; Wang et al., 1997). For example, cyanidin-3-glucoside has $\approx 75 \%$ greater antioxidant activity than cyanidin-3-galactoside (Wang et al., 1997). Furthermore, the sugar moiety may be a major determinant in the absorption of dietary flavonoids in mammals (Hollman et al., 1999; Miyazawa et al., 1999). Glucoside conjugates appear to be the most bioavailable (Hollman and Katan, 1997; Mizuma et al., 1994). The glucose transporter pathway and sodium dependent glucose receptors are thought to play a major role in absorption (Gee et al., 1998, 2000; Mizuma et al., 1994; Setchell et al., 2001; Walgren et al., 2000).

Received for publication 20 Jan. 2005. Accepted by publication 21 Mar. 2005. We thank Brian McEniry for technical assistance and Stacy Bonos and Jennifer Johnson-Cicalese for critical reading of the manuscript.

TTo whom reprint requests should be addressed.E-mail address: jpolashock@ars. usda.gov
The cultivated american cranberry (V. macrocarpon) is recognized for its brilliant red fruit due to an abundance of anthocyanins in the fruit epidermal tissues. The anthocyanins of V.macrocarpon are mainly 3-O-galactosides $(\approx 70 \%)$ and arabinosides $(\approx 24 \%)$, of cyanidin and peonidin with much lesser amounts $(\approx 3 \%$ to $9 \%)$ of glucoside anthocyanins (Hong and Wrolstad, 1990; Vogt and Jones, 2000). In contrast to V. macrocarpon, Anderson (1989) found the small-fruited cranberry ( $V$. oxycoccus $\mathrm{L}$.) to have anthocyanins consisting of mainly the glucosides of cyanidin and peonidin $(>70 \%)$, with low amounts of arabinosides $(<7 \%)$ and galactosides $(<5 \%)$.

Vaccinium oxycoccus may provide a unique opportunity to increase the relative amounts of anthocyanin glucosides in cultivated cranberry fruit through interspecific hybridization and subsequent breeding and selection. $V$. oxycoccus exists at three ploidy levels ( $2 x, 4 x$, and $6 x$ ) with $4 x$ being the most common (Camp, 1944). Previously, only tetraploid $V$. oxycoccus was available for crossing with american cranberry and the infertility inherent in heteroploid crosses resulted in limited success. Recently, diploid V. oxycoccus populations from Alaska were identified (Mahy et al., 2000) and were utilized in crosses with $V$. macrocarpon for this study. Interspecific hybrids were backcrossed to $V$. macrocarpon for introgression back into a domesticated genetic background.

The specific objectives of this study were to 1) contrast anthocyanin profiles of V.macrocarpon, with those of diploid and tetraploid $V$. oxycoccus, 2) generate a population of $V$. macrocarpon $/ V$. oxycoccus interspecifichybrids and determine their anthocyanin profiles, and 3) analyze the segregation of anthocyanin glycosylation profiles in backcross progeny (to V. macrocarpon).

\section{Materials and Methods}

Plant material. Crosses between V. macrocarpon cultivars Pilgrim, Stevens, Ben Lear, Franklin, and US93-204 and 12 diploid $V$. oxycoccus clones were made in 1998 to produce $\mathrm{F}_{1}$ hybrids, with 19 crosses using V. macrocarpon as the seed parent, and six using V. oxycoccus as the seed parent. Since the ultimate breeding 
goal was to introgress the anthocyanin glycosylation phenotype (from $V$. oxycoccus) into commercially grown, higher-yielding V. macrocarpon, $\mathrm{F}_{1}$ interspecific hybrids exhibiting vigor and greatest flower bud set were backcrossed to $V$. macrocarpon in 2000. This yielded nine backcross $\left(\mathrm{BC}_{1}\right)$ families (designated CNJ00-4, CNJ00-5, CNJ00-10, CNJ00-11, CNJ00-13, CNJ00-23, CNJ00-26, CNJ00-27, and CNJ00-29). For the initial interspecific crosses and backcrosses, pollen was collected from open flowers of the pollen parent and used fresh or stored at $4{ }^{\circ} \mathrm{C}$ in a deepwell slide until needed. Flowers to be used as the female parent were emasculated $\approx 2 \mathrm{~d}$ before anthesis. Pollen was applied to the stigma 2-3 d post-emasculation. Mature fruit were harvested and stored at $4{ }^{\circ} \mathrm{C}$ for $2-3$ months. Seeds were extracted from stored fruit, sown into 5-cm-diameter pots, and kept on a mist bench until germination. Seedlings were transplanted to $10-\mathrm{cm}$-diameter pots and grown under normal greenhouse conditions.

FrUIT ANTHOCYANIN EXTRACTION AND HIGH-PERFORMANCE LIQUID CHROMATOGRAPHY (HPLC) ANALYSIS. Fruit anthocyanins were extracted and analyzed by HPLC from representative $V$. macrocarpon cultivars ('Franklin', 'Stevens', 'Ben Lear'); seven diploid V. oxycoccus accessions (NJ96-37, NJ96-49, NJ96-76, NJ96-81, NJ96-125, NJ96-127, and NJ96-131) selected at random from a germplasm collection of native cranberry collected in Alaska (Mahy et al., 2000); three tetraploid V. oxycoccus accessions (two from Alaska, NJ96-21 and NJ96-128 and one from Clam Lake, Mich., CL20); four randomly selected $V$. macrocarpon $\mathbf{x}$ diploid V. oxycoccus $\mathrm{F}_{1}$ hybrids ('Franklin' x NJ96-81, 'Pilgrim' x NJ96-81, 'Ben Lear' x NJ 96-127, and NJ96-81 x 'Franklin'); and 118 randomly selected progeny from the nine backcross families described above.

Fruit were harvested when ripened to a moderate to dark red color and placed in a freezer at $-20{ }^{\circ} \mathrm{C}$. Approximately $2 \mathrm{~g}$ of ripe berries from each plant were ground in $20 \mathrm{~mL}$ of $1 \% \mathrm{HCl}$ in methanol (isolation solution). Progeny with $<2 \mathrm{~g}$ fruit samples were extracted with 5-10 mL of isolation solution. Samples were filtered through two layers of cheesecloth and centrifuged for $10 \mathrm{~min}$ at $3000 \mathrm{~g}_{\mathrm{n}}$. The supernatant was dried to completion in a Rotavapor (Brinkmann Instruments, Westbury, N.Y.) and resuspended in a small volume $(500 \mu \mathrm{L})$ of isolation solution. Extracts were centrifuged at $11,000 g_{n}$ for 1 min to pellet any insoluble material and the supernatant was stored at $4^{\circ} \mathrm{C}$. Extracts were centrifuged one more time at $3000 g_{n}$ through a $0.45-\mu \mathrm{m}$ nylon filter (Corning Costar, Corning, N.Y.) immediately before injection. The anthocyanins were characterized by HPLC model DX-500 with a PDA-100 variable diode array detector (Dionex Corp., Sunnyvale, Calif.) on a $4.6 \times 250-\mathrm{mm}, 5-\mu \mathrm{m}$ Zorbax SBC18 column (Agilent Technologies, PaloAlto, Calif.). Separations were carried out using a 20-min linear gradient of $0 \%$ to $20 \%$ (v/v) solvent A (100\% acetonitrile) with the balance being solvent B $(1.5 \%(\mathrm{v} / \mathrm{v})$ phosphoric acid and $15 \%(\mathrm{v} / \mathrm{v})$ acetic acid in water), followed by a 2 -min hold at $20 \%$ solvent A. Injection volume was $20 \mu \mathrm{L}$ with a flow rate of $1.0 \mathrm{~mL} \cdot \mathrm{min}^{-1}$ and detection was by absorption at $520 \mathrm{~nm}$. Retention times were confirmed with available standards of cyanidin-3-galactoside, cyanidin-3-glucoside, and peonidin-3-glucoside (Extrasynthese, Genay, France), and by comparison to the established anthocyanin profile for cranberry (Anderson, 1989; Vorsa et al., 2003).

Statistical ANAlysis. Chi-square goodness-of-fit test utilized Yates correction factor to test for fit to a single locus model (Zar, 1974).

\section{Results}

SPECIES ANTHOCYANIN PROFILES. Fruit anthocyanin profiles of the V. macrocarpon cultivars sampled were consistent with those previously reported (Vorsa et al., 2003), with $<7 \%$ of the anthocyanins glucosylated (Table 1). The anthocyanin profile of fruit from the cultivar Stevens is representative of V.macrocarpon (Fig. 1A), showing the anthocyanin peaks cyanidin-3-galactoside (Rt 6.0-6.5 min), cyanidin-3-glucoside (Rt 7.0-7.5 min), cyanidin-3-arabinoside (Rt 8.0-8.5 min), peonidin-3-galactoside (Rt 9.0-9.5 min), peonidin-3-glucoside (Rt 10.5-11.0), and peonidin3-arabinoside (11.5-12.0 min). Total galactosides ranged from $63 \%$ to $69 \%$ and total arabinosides ranged from $27 \%$ to $33 \%$. The small-fruited cranberry (V.oxycoccus) exhibited two distinct profiles, which were associated with ploidy level. All tetraploid V. oxycoccus samples exhibited an anthocyanin profile (Fig. 1B) similar to that of $V$. macrocarpon with principally galactosides and arabinosides of cyanidin and peonidin (Table 1). However, tetraploid $V$. oxycoccus exhibited lower levels of galactosides (49\% to $52 \%$ ) and higher levels of arabinosides (39\% to $48 \%$ ) in comparison to $V$. macrocarpon.

In contrast to V.macrocarpon and tetraploid V.oxycoccus, fruit anthocyanins of diploid $V$. oxycoccus were principally glucosides of cyanidin and peonidin (Fig. $1 \mathrm{C}$ and D; Table 1). Cyanidin-3glucoside ranged from $\approx 13 \%$ to $29 \%$ and peonidin-3-glucoside ranged from $\approx 42 \%$ to $70 \%$, based on percent peak area (Table 1 ). Total anthocyanin glucosides of diploid $V$. oxycoccus fruit were $\approx 74 \%$. The next most abundant anthocyanin conjugate was arabinoside, with cyanidin-3-arabinoside ranging from $6 \%$ to $20 \%$ and peonidin-3-arabinoside ranging from $1 \%$ to $26 \%$ (Table 1 ). The total arabinosides varied from $15 \%$ to $40 \%$ (Fig. $1 \mathrm{C}$ and D). Galactosides of cyanidin and peonidin together totaled $<4 \%$.

INTERSPECIFIC HYBRID ANTHOCYANIN PROFILES. Fruit from the $F_{1}$ hybrids exhibited the six major anthocyanins (Fig. 2; Table 1) discussed above. The cyanidin and peonidin derivatives were in similar proportions, with $\approx 45 \%$ cyanidin and $\approx 55 \%$ peonidin glycosides. The total glucosides ranged from $37 \%$ to $49 \%$, galactosides from $35 \%$ to $39 \%$, and arabinosides from $16 \%$ to $24 \%$. The proportions of the glucosides and galactosides were intermediate between those of the two parental species, however, the percentage of arabinosides was lower than might be expected (i.e., it was lower in the hybrids than either of the two parents). All hybrid progeny had a very similar profile regardless of maternal parent species. In some $F_{1}$ hybrids, derivatives of delphinidin with retention times consistent with delphinidin galactoside (Rt4.5-5.0 $\mathrm{min}$ ) and delphindin glucoside (Rt 5.0-5.5 min) were apparent, but accounted for $<1 \%$ of the total anthocyanins.

BACKCROSS-1 ANTHOCYANIN PROFILES. The percent of anthocyanin glucosides ranged from $2.5 \%$ to $70 \%$ in fruit of the first backcross (to $V$. macrocarpon) progeny and exhibited a bimodal frequency distribution(Fig. 3, glucosides). Percent of anthocyanin galactosides ranged from $18.6 \%$ to $78.5 \%$, also exhibiting a bimodal frequency distribution (Fig. 3, galactosides). Arabinosides ranged from $6.5 \%$ to $60 \%$, with a unimodal distribution (Fig. 3, arabinosides). In a $V$. macrocarpon germplasm survey of over 250 cultivars, the lowest galactoside proportion found was $63 \%$ (Vorsa et al., 2003). With a single locus model, one would expect $50 \%$ of the progeny to have a $V$. macrocarpon phenotype ( $>63 \%$ anthocyanin galactosides) and $50 \%$ of the progeny would be expected to have a profile similar to that of the $\mathrm{F}_{1}$ hybrids $(<63 \%$ anthocyanin galactosides) where the locus would be heterozygous. Thus, the backcross progeny can be categorized into one of two 
Table 1. Mean percent of the six major ${ }^{\mathrm{z}}$ fruit anthocyanins in cranberry for $V$. macrocarpon, $V$. oxycoccus $(2 x)$, V. oxycoccus (4x), and V. macrocarpon $\mathrm{x}$ V. oxycoccus $(2 x)$ hybrids.

\begin{tabular}{|c|c|c|c|c|c|c|c|}
\hline \multirow[b]{2}{*}{ Population } & \multirow[b]{2}{*}{$\mathrm{N}$} & \multicolumn{6}{|c|}{ Mean \pm SD of the six major anthocyanins $(\%)$} \\
\hline & & Cy-3-galy & Cy-3-glu & Cy-3-arab & Pn-3-gal & Pn-3-glu & Pn-3-arab \\
\hline \multirow[t]{2}{*}{ V. macrocarpon } & 3 & $32.2 \pm 5.7$ & $1.3 \pm 0.9$ & $19.2 \pm 4.4$ & $33.4 \pm 6.5$ & $3.7 \pm 0.8$ & $10.2 \pm 2.8$ \\
\hline & & $26.9-37.7 x$ & $0.7-2.6$ & $14.0-23.1$ & $27.0-41.8$ & $2.7-4.5$ & $7.1-14.0$ \\
\hline \multirow[t]{2}{*}{ V. oxycoccus $(2 x)$} & 7 & $0.5 \pm 0.7$ & $19.5 \pm 6.5$ & $13.1 \pm 4.8$ & $0.4 \pm 0.8$ & $54.5 \pm 9.6$ & $12.0 \pm 8.3$ \\
\hline & & $0-1.5$ & $12.7-29.4$ & $6.3-19.5$ & $0-2.2$ & $42.3-69.6$ & $1.2-26.0$ \\
\hline \multirow[t]{2}{*}{ V. oxycoccus $(4 x)$} & 3 & $26.7 \pm 6.1$ & $1.5 \pm 1.1$ & $25.3 \pm 2.7$ & $24.5 \pm 4.2$ & $4.7 \pm 2.1$ & $17.3 \pm 7.7$ \\
\hline & & $19.7-31.0$ & $0.5-2.6$ & $22.4-27.8$ & $21.3-29.3$ & $2.4-6.5$ & $10.8-25.7$ \\
\hline \multirow[t]{2}{*}{ Hybridsw } & 4 & $18.3 \pm 2.4$ & $13.2 \pm 3.0$ & $13.0 \pm 1.9$ & $18.2 \pm 2.0$ & $29.0 \pm 3.4$ & $8.4 \pm 2.6$ \\
\hline & & $15.0-20.8$ & $9.8-17.0$ & $11.2-15.2$ & $16.2-20.9$ & $24.4-31.8$ & $5.2-11.5$ \\
\hline
\end{tabular}

zThese six anthocyanins represent over $95 \%$ of the total anthocyanins in all four populations.

y Cy-3-gal (cyanidin-3-O-galactoside), Cy-3-glu (cyanidin-3-O-glucoside), Cy-3-arab (cyanidin-3-O-arabinoside), Pn-3-gal (peonidin3-O-galactoside), Pn-3-glu (peonidin-3-O-glucoside), and Pn-3-arab (Peonidin-3-O-arabinoside).

xRanges (\%).

${ }^{\mathrm{w}} \mathrm{V}$. macrocarpon $\mathrm{x} V$. oxycoccus $(2 x)$ and reciprocal.

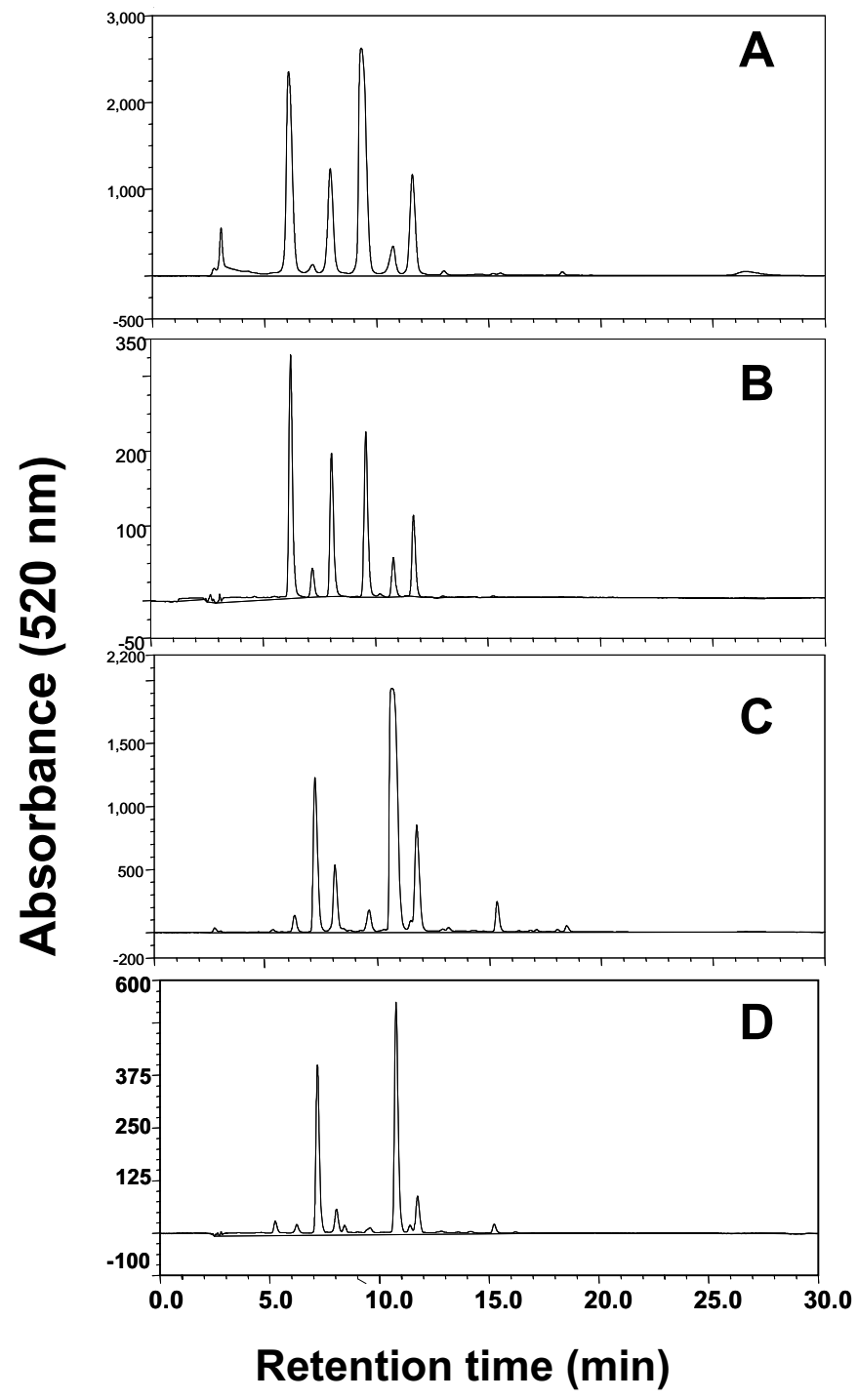

Fig. 1. Representative HPLC anthocyanin profiles from fruit for: V. macrocarpon, cv. Stevens (A); tetraploid V. oxycoccus CL-20 (B), and diploid V. oxycoccus accessions NJ96-49 (C), and NJ96-76 (D). Retention time (Rt) for anthocyanins are: cyanidin-3-galactoside (Rt 6.0-6.5 min), cyanidin-3-glucoside (Rt 7.0-7.5 $\mathrm{min}$ ), cyanidin-3-arabinoside (Rt 8.0-8.5 min), peonidin-3-galactoside (Rt 9.0-9.5 min), peonidin-3-glucoside (Rt 10.5-11.0 min), and peonidin-3arabinoside (Rt 11.5-12.0 min).

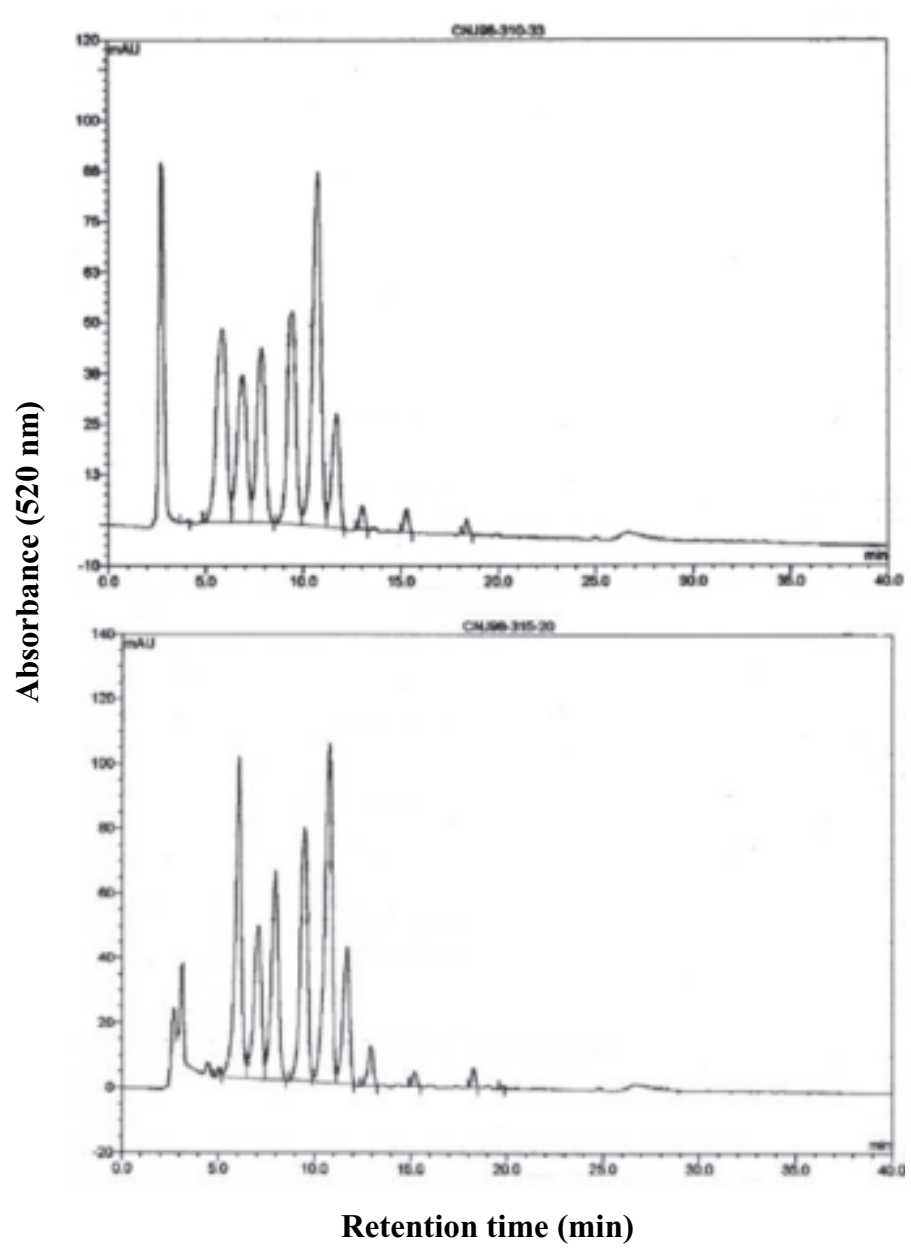

Fig. 2. Representative HPLC anthocyanin profiles of fruit from two representative $\mathrm{F}_{1}$ V. macrocarpon $\mathrm{x}$ V. oxycoccus hybrid progeny. 


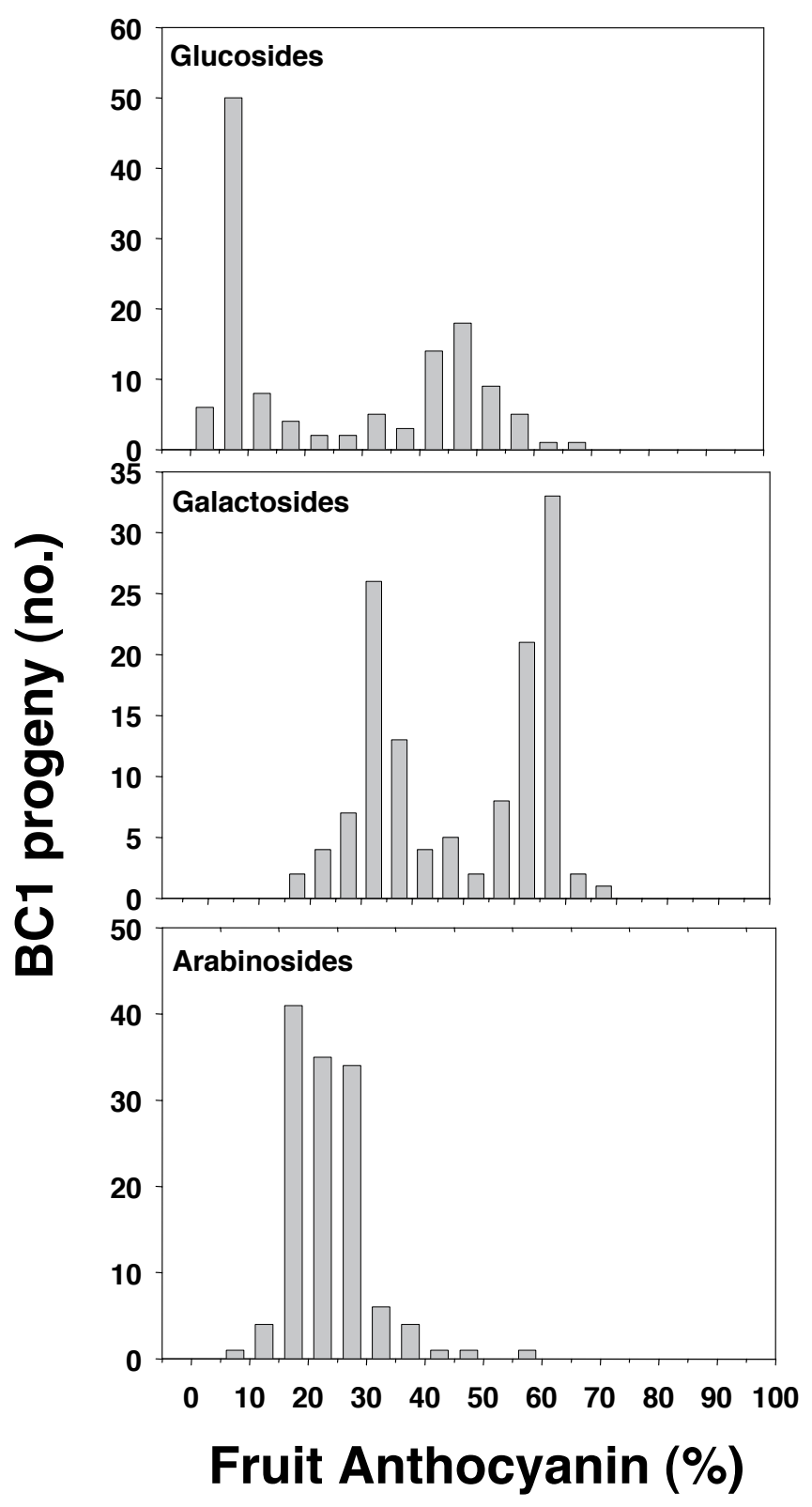

Fig. 3. Frequency distribution of percent fruit anthocyanin (cyanidin and peonidin) glucosides, galactosides and arabinosides in backcross $[(V$. macrocarpon $\mathrm{x}$ $V$. oxycoccus) $\times$ V. macrocarpon] progeny. Classes are represented by $5 \%$ groupings, $\mathrm{n}=118$.

classes based on percent anthocyanin galactosides ( $>63 \%$ and $<63 \%$ ), and tested for fit to a single locus model. The frequency distribution (42:76) deviated significantly from a Mendelian one locus model $\left(\chi^{2}=9.2\right.$; df $\left.=1 ; P<0.005\right)$, with fewer progeny than expected having a galactoside proportion greater than $63 \%$. Utilizing percent glucosylation as the criterion, with $8 \%$ glucosides as maximum for the $V$. macrocarpon phenotype based on a germplasm survey (Vorsa et al., 2003), the frequency distribution deviated significantly from a Mendelian one locus model $\left(\chi^{2}=\right.$ 9.6 , df $=1 ; P<0.005$ ). Transgressive phenotypes (35 progeny, or $27.3 \%$ ) were recovered, as defined by backcross phenotypes with less than $35 \%$ galactosides (lower limit for galactosides in $\mathrm{F}_{1}$ hybrids), and individuals with the glucoside classes between $8 \%$ (upper limit of $\mathrm{V}$. macrocarpon) and $35 \%$ (lower limit of $\mathrm{F}_{1}$ hybrid distribution).

\section{Discussion}

In both cranberry species, V. macrocarpon and V. oxycoccus, all three sugar conjugates (galactosides, glucosides, and arabinosides) are present in fruit anthocyanins. However, the proportions of galactosides versus glucosides of the anthocyanidins cyanidin and peonidin vary considerably between these two species. Galactosides are the predominant form in $V$. macrocarpon $(>60 \%)$, whereas glucosides are the predominant form in diploid V. oxycoccus $(>70 \%)$ as reported for this species by Anderson (1989). The bimodal distribution of the backcross progeny suggests a major locus exists determining whether the anthocyanins are conjugated to either galactose or glucose. However, the occurrence of phenotypes intermediate to the $V$. macrocarpon ( $>63 \%$ galactosides) and $\mathrm{F}_{1}$ hybrid ( $<40 \%$ galactosides) phenotypes indicates that the glycosylation difference between these two cranberry species is likely controlled by more than one locus. Similarly, the backcross populations yielded individuals with glucosylation classes outside the ranges of the parental species and the first generation hybrids, suggesting the presence of quantitative modifying loci.

In a number of plant species, anthocyanidins are conjugated to glucose by a UDP-glucose:flavonoid 3-O-glucosyltransferase to form anthocyanidin 3-O-glucosides (Holton and Cornish, 1995). Glycosyltransferases are considered to be members of a large supergene family having broad affinity for substrates, but with both regioselectivity and regiospecificity for the sugar acceptor (Vogt and Jones, 2000). An UDP-glucose:flavonoid-3-O-glucosyltransferase isolated from Vitis vinifera $\mathrm{L}$. did not conjugate UDPgalactose (Ford et al., 1998). Furthermore, in species producing predominately 3-O-galactosylated flavonols, 3-O-glucosylated flavonols can be substantially increased with transformation and expression of UDP-glucose:flavonoid-3-O-glucosyltransferase (Schwinn et al., 1997). The difference in anthocyanin glycosylation between these two cranberry species could be a function of different anthocyanin 3-O-glycosyltransferase alleles. Thus, it could be hypothesized that $V$. macrocarpon is fixed for an allele that conjugates predominantly galactose and diploid $V$. oxycoccus is fixed for an allele that conjugates predominately glucose. However, Vogt and Jones (2000) argue that the supply and quality of nucleotides (e.g., UDP-glucose etc.) would be highly conserved during evolution leading to high substrate specificity for the sugar donor. Since both species also produce fruit anthocyanin 3 - $O$-arabinosides in relatively high $(>15 \%)$ abundance, as well as in all segregating populations, it would suggest that there is a second locus for a 3-O-arabinotransferase in both species. Thus, in heterozygotes there could be three distinct glycosyltransferases competing for the aglycone substrate pool.

The conjugation for another flavonoid class, flavonols, in cranberry appears to be similar between these two species, with quercetin 3-O-galactoside being the major sugar conjugate (I. Vvedenskaya and N. Vorsa, unpublished data). This suggests that in cranberry, flavonols are conjugated by a different set of 3-O transferases specific to flavonols. Evidence for maternal effects was not found, suggesting that fruit anthocyanin glycosylation profiles are nuclear encoded and controlled.

In conclusion, we have confirmed that the $V$. macrocarpon anthocyanin fruit profile is fairly uniform amongst the cultivars tested and consists of six major anthocyanins, i.e., the galactosides, glucosides and arabinosides of cyanidin and peonidin. These results are similar to those published for this species (Sapers and Hargrave, 1987). The anthocyanin fruit profiles for $V$. oxycoccus, although having the same six major anthocyanins, differed 
at the two ploidy levels tested. The tetraploid accessions had a profile more similar to that of V. macrocarpon, while the diploid V. oxycoccus profile was quite different with very high levels of glucosides. Genetic evidence, based on isozyme analysis, suggests tetraploid $V$. oxycoccus has been introgressed with $V$. macrocarpon or has been derived from an ancestral diploid species other than the diploid V. oxycoccus of this study (Mahy et al., 2003). The anthocyanin data also support this hypothesis. The diploid $V$. oxycoccus anthocyanin profiles of this study were similar to those published by Anderson (1989).

The first generation hybrids ( $V$. macrocarpon $\mathrm{x}$ diploid $V$. oxycoccus) were intermediate for most morphological and phenological traits (data not shown) as well as for the anthocyanin fruit profiles. Specifically, the high anthocyanin galactosides and arabinosides typical of $V$. macrocarpon were reduced with a dramatic increase of glucosides, which is typical of diploid $V$. oxycoccus. Segregation in the backcross population suggests that this is a viable approach to increasing the proportion of glucosylated anthocyanins in cultivated $V$. macrocarpon with a potential improvement in antioxidant bioavailability.

\section{Literature Cited}

Anderson, O. 1989. Anthocyanins in fruits of Vaccinium oxycoccus L. (small cranberry). J. Food Sci. 54:383-384.

Benvenuti, S., F. Pellati, M. Melegari, and D. Bertelli. 2004. Polyphenols, anthocyanins, ascorbic acid, and radical scavenging activity of Rubus, Ribes, and Aronia. J. Food Sci. 69:164-169.

Camp, W.H. 1944. A preliminary consideration of the biosystematy of Oxycoccus. Bul. Torrey Bot. Club 71:426-437.

Cao, G., E. Sofic, and R.L. Prior. 1997. Antioxidant and prooxidant behavior of flavonoids: Structure-activity relationships. Free Radical Biol. Medicine 22:749-760.

Foo, L., Y. Lu, A.B. Howell, and N. Vorsa. 2000a. A-Type proanthocyanidin trimers from cranberry that inhibit adherence of uropathogenic P-fimbriated Escherichia coli. J. Natural Products 63:1225-1228.

Foo, L., Y. Lu, A.B. Howell, and N. Vorsa. 2000b. The structure of cranberry proanthocyanidins which inhibit adherence of uropathogenic P-fimbriated Escherichia coli in vitro. Phytochemistry 54:173-181.

Ford, C.M., P.K. Boss, and P.B. Hoj. 1998. Cloning and characterization of Vitis vinifera udp-glucose:flavonoid 3-o-glucosyltransferase, a homologue of the enzyme encoded by the maize bronze- 1 locus that may primarily serve to glucosylate anthocyanidins in vivo. J. Biol. Chem. 273:9224-9233.

Gee, J.M., M.S. DuPont, A.J. Day, G.W. Plumb, G. Williamson, and I.T. Johnson. 2000. Intestinal transport of quercetin glycosides in rats involves both deglycosylation and interaction with the hexose transport pathway. J. Nutr. 130:2765-2771.

Gee, J.M., M.S. DuPont, M.J. Rhodes, and I.T. Johnson. 1998. Quercetin glucosides interact with the intestinal glucose transport pathway. Free Radical Biol. Medicine 25:19-25.

Hollman, P.C., M.N. Bijsman, Y. van Gameren, E.P. Cnossen, J.H. deVries, and M.B. Katan. 1999. The sugar moiety is a major determinant of the absorption of dietary flavonoid glycosides in man. Free Radical Res. 31:569-573.
Hollman, P.C.H. and M.B. Katan. 1997. Absorption, metabolism and health effects of dietary flavonoids in man. Biomedicine Pharmacotherapy 51:305-310.

Holton, T.A. and E.C. Cornish. 1995. Genetics and biochemistry of anthocyanin biosynthesis. Plant Cell 7:1071-1083.

Hong, V. and R.E. Wrolstad. 1990. Use of HPLC separation/photodiode array detection for characterization of anthocyanins. J. Agr. Food Chem. 38:708-715.

Koes, R.E., F. Quattrocchio, and J.N.M. Mol. 1994. The flavonoid biosynthetic pathway in plants: Function and evolution. BioEssays 16:123-132.

Mahy, G., L.P. Bruederle, B. Connors, M.V. Hofwegen, and N. Vorsa. 2000. Allozyme evidence for genetic autopolyploidy and high genetic diversity in tetraploid cranberry, Vaccinium oxycoccus (Ericaceae). Amer. J. Bot. 87:1882-1889.

Miyazawa, T., K. Nakagawa, M. Kudo, K. Muraishi, and K. Someya. 1999. Direct intestinal absorption of red fruit anthocyanins, cyanidin3-glucoside and cyanidin-3,5-diglucoside, into rats and humans. J. Agr. Food Chem. 47:1083-1091.

Mizuma, T., K. Ohta, and S. Awazu. 1994. The $\beta$-anomeric and glucose preferences of glucose transport carrier for intestinal active absorption of monosaccharide conjugates. Biochim. Biophys. Acta 1200:117-122.

Nijveldt, R.J., E. van Nood, D.E.C. van Hoorn, P.G. Boelens, K. van Norren, and P.A.M. van Leeuwen. 2001. Flavonoids: A review of probable mechanisms of action and potential applications. Amer. J. Clinical Nutr. 74:418-425.

Sapers, G. and D. Hargrave. 1987. Proportions of individual anthocyanins in fruits of cranberry cultivars. J. Amer. Soc. Hort. Sci. 112:100-104.

Satue-Gracia, M.T., M. Heinonen, and E.N. Frankel. 1997. Anthocyanins as antioxidants on human low-density lipoprotein and lecithin-liposome systems. J. Agr. Food Chem. 45:3362-3367.

Schijlen, E.G.W.M., C.H. R.deVos, A.J. van Tunen, and A.G. Bovy. 2004. Modification of flavonoid biosynthesis in crop plants. Phytochemistry 65:2631-2648.

Schwinn, K.E., K.M. Davies, S.C. Deroles, K.R. Markham, R.M. Miller, J.M. Bradley, D.G. Manson, and N.K. Given. 1997. Expression of an Antirrhinum majus UDP-glucose:flavonoid-3-O-glucosyltransferase alters flavonoid glycosylation and acylation in lisianthus (Eustoma grandiflorum Grise). Plant Sci. 125:53-61.

Setchell, K., N. Brown, P. Desai, L. Zimmer-Nechemais, B. Wolf, W. Brashear, A. Kirschner, A. Cassidy, and J. Heubi. 2001. Bioavailability of pure isoflavones in healthy humans and analysis of commercial soy isoflavone supplements. J. Nutr. 131:1362S-1375S.

Vogt, T. and P. Jones. 2000. Glycosyltransferases in plant natural product synthesis: Characterization of a supergene family. Trends Plant Sci. 5:380-386.

Vorsa, N., J. Polashock, D. Cunningham, and R. Roderick. 2003. Genetic inferences and breeding implications from analysis of cranberry germplasm anthocyanin profiles. J. Amer. Soc. Hort. Sci. 128:691-697.

Walgren, R.A., J.-T. Lin, R.K.-H. Kinne, and T. Walle. 2000. Cellular uptake of dietary flavonoid quercetin 4-B-Glucoside by sodium-dependent glucose transporter SGLT1. J. Pharmacology Expt. Therapeutics 294:837-843.

Wang, H., G. Cao, and R.L. Prior. 1997. Oxygen radical absorbing capacity of anthocyanins. J. Agr. Food Chem. 45:304-309.

Zar, J. 1974. Biostatistical analysis. Prentice Hall, Englewood Cliffs, N.J. 